\title{
Three-dimensional printed miniaturized spectral system for collagen fluorescence lifetime measurements
}

Luwei Zou

Ronald Koslakiewicz

Mohamad Mahmoud

Mehdi Fahs

Rui Liu

Joe Fujiou Lo 


\title{
Three-dimensional printed miniaturized spectral system for collagen fluorescence lifetime measurements
}

\author{
Luwei Zou, Ronald Koslakiewicz, Mohamad Mahmoud, Mehdi Fahs, Rui Liu, and Joe Fujiou Lo* \\ University of Michigan-Dearborn, Department of Mechanical Engineering, Bioengineering Program, 2088 IAVS Building, \\ 4901 Evergreen Road, Dearborn, Michigan 48128, United States
}

\begin{abstract}
Various types of collagens, e.g., type I and III, represent the main load-bearing components in biological tissues. Their composition changes during processes such as wound healing and fibrosis. When excited by ultraviolet light, collagens exhibit autofluorescence distinguishable by their unique fluorescent lifetimes across a range of emission wavelengths. Here, we designed a miniaturized spectral-lifetime detection system as a noninvasive probe for monitoring tissue collagen compositions. A sine-modulated LED illumination was applied to enable frequency domain fluorescence lifetime measurements under three wavelength bands, separated via a series of longpass dichroics at 387, 409, and $435 \mathrm{~nm}$. We employed a lithography-based three-dimensional (3-D) printer with $<50 \mu \mathrm{m}$ resolution to create a custom designed optomechanics in a handheld form factor. We examined the characteristics of the optomechanics with finite element modeling to simulate the effect of thermal (from LED) and mechanical (from handling) strain on the optical system. The geometry was further optimized with ray tracing to form the final 3-D printed structure. Using this device, the phase shift and demodulation of collagen types were measured, where the separate spectral bands enhanced the differentiation of their lifetimes. This system represents a low cost, handheld probe for clinical tissue monitoring applications. ๑ 2016 Society of Photo-Optical Instrumentation Engineers (SPIE) [DOI: 10.1117/1.JBO.21.7.075001]
\end{abstract}

Keywords: spectral-lifetime; collagen; frequency domain; three-dimensional printing.

Paper 160173PR received Mar. 19, 2016; accepted for publication Jun. 13, 2016; published online Jul. 1, 2016.

\section{Introduction}

Collagen composition in the tissue's extracellular matrix (ECM) varies during many processes such as wound healing, fibrosis, and aging. In wound healing, specifically, ECM is disrupted by the act of wounding and injury. In addition, the proliferation and remodeling phase of wound healing involves a change from immature type III collagen to the stronger type I collagen. ${ }^{1-4}$ Monitoring collagen transition during these processes, then, can provide important information about wound maturity and strength. Current techniques to visualize and quantify changes in tissue collagen types rely on immunohistochemistry and polarized picrosirius staining under the microscope. ${ }^{5-8}$ Unique methods such as laser-induced fluorescence and multiphoton excitation are becoming popular as label-free detection techniques, which have already been applied to ocular, skin, and liver tissue microscopies, ${ }^{9-17}$ in addition to our previous work on differentiating collagen types via fluorescence lifetimes. ${ }^{18}$ However, these aforementioned techniques are not designed for clinical use, and their bulk and complexity reduce their usability in applications such as wound monitoring and noninvasive detections. Motivated by the benefits and limitation of noninvasive techniques, we designed a compact, low-cost optical system based on frequency domain fluorescence lifetimes to measure tissue collagen (Fig. 1). Three dimensional (3-D) printing was employed to prototype such miniature optical systems, while the optical components including dichroic, lenses, and filters were selected with a diameter of 0.5 in., creating an overall cylindrical device dimension of $\sim 1.5$ in. $\times 3$ in.. Thermal and mechanical characteristics of the 3-D printed optomechanics were analyzed using finite element analysis (FEA) and found to have minimal effects on ray tracing. Sine-modulated excitation and spectrally separated frequency domain emissions were then digitized and analyzed in MATLAB ${ }^{\circledR}$ to calculate their lifetimes. Our results showed that such compact, spectral-lifetime detection system balances the portability with specificity for differentiating collagen types, in a package that is suitable for tissue monitoring applications.

\subsection{Collagen Spectral Lifetime}

Autofluorescence from collagen is commonly observed in fluorescence microscopy. The primary fluorophores contributing to collagen's fluorescence are pyridinium, tyrosine, and phenylalanine groups,${ }^{19-22}$ which can be affected by crosslinking, glycation, and their compositions in different types of collagens. ${ }^{23-25}$ The effects of these multiple fluorophores can be seen in the lifetime of the collagen fluorescence, with evidence that lifetime also varies across the emission spectrum. ${ }^{26-28}$ Spectral-lifetime detection, then, can help distinguish the compositions of collagen types in a mixed environment such as tissue or the skin. A simplified model of spectral-lifetime fluorescence can be described as

$$
I(\lambda, t)=\sum_{i=1} \alpha_{i}(\lambda) e^{-\frac{t}{\tau_{i}}}
$$



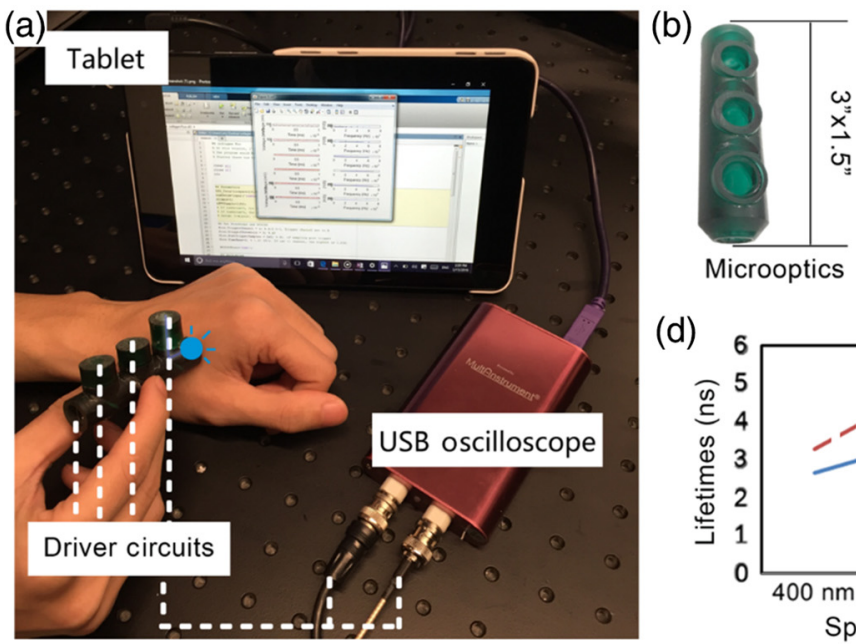

(c)

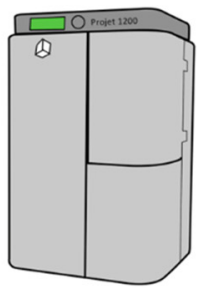

(d)

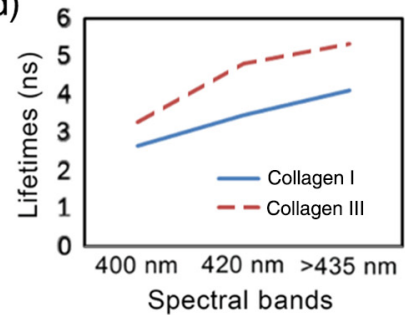

Fig. 1 Schematics of the 3-D printed probe for tissue collagen differentiation. (a) The prototype system is comprised of the micro-optics system, LED, photodiode, driver electronics, USB oscilloscope, and a tablet for data analysis. It is designed to measure tissue collagen compositions via frequency domain fluorescence detections. (b) A $3 \mathrm{in} . \times 1.5 \mathrm{in}$. prototype micro-optics mount was enabled by (c) 3-D stereolithogrpahy printing with $<50-\mu \mathrm{m}$ resolution. (d) By leveraging both spectral and lifetime spectroscopy, the prototype can provide better differentiation of the different types of collagen, e.g., I and III.

where the values of $\alpha_{i}(\lambda)$ represent the relative contribution of the $i$ 'th fluorophore at wavelength $\lambda$. The frequency response is typically measured at several wavelengths resulting in wavelength-dependent phase angle and the modulation values. ${ }^{29}$

We observed that under the same illumination, collagens have varying fluorescence lifetimes corresponding to different wavelength bands. ${ }^{4}$ We hypothesize that under these conditions there will be certain wavelength bands that augment the fluorescence lifetime separation of different collagens. To investigate this possibility, we divided the fluorescence emission into three channels using three dichroics at 387, 409, and $435 \mathrm{~nm}$. For wound healing applications, tissue collagen composition changes from type III to type I. The aforementioned bands cover the peak, shoulder, and tail portion of the autofluorescence spectra of both collagens.

\subsection{Optomechanics Rapid Prototyping Via Additive Manufacturing}

Additive manufacturing has been widely popularized for rapid prototyping in recent works, including 3-D printed optical systems. Despite the limited spatial resolution and plastic built material in current commercial printers, there are already efforts in testing and characterizing 3-D printing for optical systems and optomechanical components. ${ }^{30}$ Other optics and photonics applications include structured 3-D phantoms, ${ }^{31}$ optofluidics, ${ }^{32}$ as well as miniaturized microscopy, ${ }^{33}$ in addition to numerous bioengineering applications. However, a high-resolution printer is necessary in our micro-optics design, where half inch optics need to be precisely aligned in a miniaturized setup. A $1^{\circ}$ change in the dichoric orientation, e.g., would incur a focal change on the order of $400 \mu \mathrm{m}$, which is a significant portion of the 1.5mm-diameter photodiode used in this micro-optic system. To address this, a digital light processing (DLP)-based lithography 3 -D printer with $43-\mu \mathrm{m}$ resolution is employed to prototype the micro-optics. DLP lithography is a 3-D printing method that uses an ultraviolet light to photo-crosslink a liquid polymer substrate. ${ }^{34}$ Models built with CAD drawing program can be easily inserted into the software for the 3-D printer. This compact professional printer can give us 585-dpi print resolution or $43-\mu \mathrm{m}$ resolution, with a layer thickness of $30 \mu \mathrm{m}$. Unlike an extrusion-based method where models are printed line-byline, DLP 3-D printing is imaged layer-by-layer, and the resolution does not affect the print speed. Only several hours of printing, without machining, are required to prototype our device with complex internal structure within those geometric accuracies.

\subsection{Frequency Domain Lifetime}

Fluorescence from biomolecules, such as tissue collagens, can be modeled as a decay event with a characteristic impulse response function specific to particular fluorophores. This impulse response function can be measured in time domain using a short excitation pulse, or using a time-varying sinusoidal intensity in frequency domain. ${ }^{18,35,36}$ The frequency domain method preserves the modulation frequency of the sinusoid, while changing the modulation amplitude and phase as a function of the specific fluorophore's impulse response decay. This is analogous to a linear time-invariant system encountered in electrical circuits. Considering the portable nature of our application, frequency domain technique is a lower complexity, costeffective alternative for monitoring biological tissues compared to time-domain method. ${ }^{37,38}$ Optimum modulation frequencies for tissue structural proteins such as collagens and elastin range from 10 to $100 \mathrm{MHz} .{ }^{39}$ This lowers the requirements for the LED driver electronics, photodetector amplifier responses, and signal digitization and recording speeds. ${ }^{40,41} \mathrm{~A}$ single, portable USB multichannel digital storage oscilloscope (DSO) can perform the acquisitions for both the excitation and emissions waveforms. Using the phase $\Delta \phi$ and modulation $m$ from oscilloscope measurements, two lifetimes can be calculated and combined via multiexponential models, at particular wavelengths, to find characteristic lifetimes of a sample. 


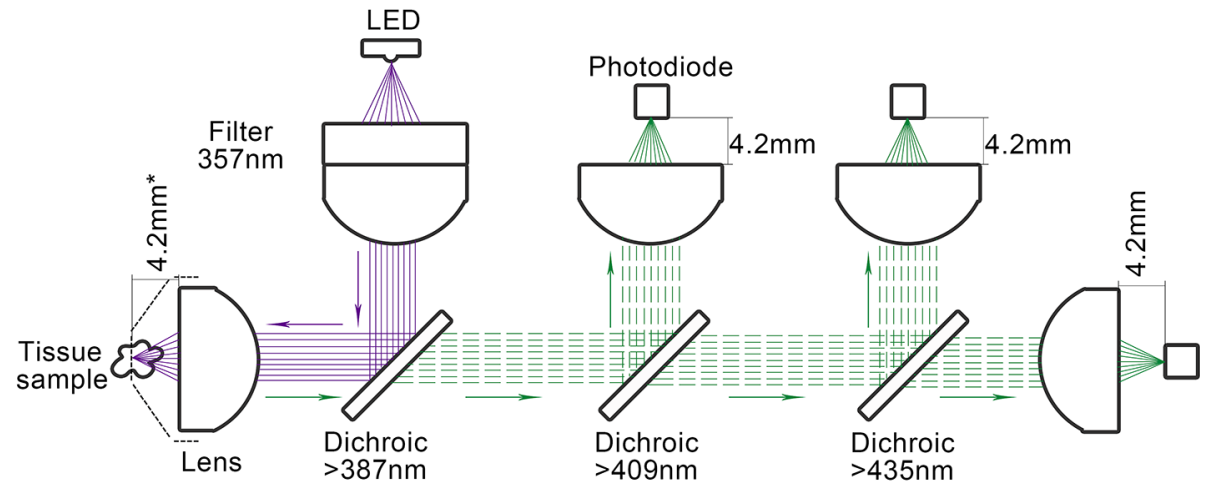

* Geometry fixed by hollow cavity structure

Fig. 2 Spectral-lifetime micro-optics system setup. Three 45 deg longpass dichorics at 387,409 , and $435 \mathrm{~nm}$ separate the system into four stages. All lenses were designed as $f=4.2 \mathrm{~mm}$ condenser lenses to simplify the design. The first stage filters and collimates the 357-nm LED light onto the dichroic and subsequently focuses it on a fluorescence sample, e.g., skin, at a fixed geometry described by the lens' focal length and a cavity within the 3-D printed mount. Emission and scattering are collected through the same exact optical axis to maximize coupling, while the $387-\mathrm{nm}$ dichroic removes the scattering intensities.

Table 1 Component list of the system.

\begin{tabular}{|c|c|c|c|c|c|c|}
\hline Components & Type & Part & Specifications & Diameter & Volt & Price $(\$)$ \\
\hline \multicolumn{7}{|c|}{ Sample optical coupling } \\
\hline Lenses & Condenser & ACL108 & $f 4.2 \mathrm{~mm}$ & $10 \mathrm{~mm}$ & & 16.8 \\
\hline \multicolumn{7}{|c|}{ Excitation } \\
\hline Optoelectronics & LED & Digikey 537-1092 & $\lambda 365 \mathrm{~nm}$ & $4.4 \mathrm{~mm}$ & $4.1 \mathrm{~V}$ & 35.75 \\
\hline Lenses & Condenser & ACL108 & $f 4.2 \mathrm{~mm}$ & $10 \mathrm{~mm}$ & & 16.8 \\
\hline Filter & Band pass & Edmund 86-973 & $\lambda 357 \mathrm{~nm}$ & $12.5 \mathrm{~mm}$ & & 180 \\
\hline Dichroic & Longpass & Chroma T387Ip & $\lambda 387 \mathrm{~nm}$ & $12.5 \mathrm{~mm}$ & & 300 \\
\hline \multicolumn{7}{|c|}{$400 \mathrm{~nm}$ band } \\
\hline Optoelectronics & Photodiode & Thor FDS025 & $\lambda 400-1100 \mathrm{~nm}$ & $5.4 \mathrm{~mm}$ & $20 \mathrm{~V}$ & 30 \\
\hline Lenses & Condenser & ACL108 & $f 4.2 \mathrm{~mm}$ & $10 \mathrm{~mm}$ & & 16.8 \\
\hline Dichroic & Longpass & Edmund 86-309 & $\lambda 409 \mathrm{~nm}$ & $12.5 \mathrm{~mm}$ & & 140 \\
\hline \multicolumn{7}{|c|}{$420 \mathrm{~nm}$ band } \\
\hline Optoelectronics & Photodiode & Thor FDS025 & $\lambda 400-1100 \mathrm{~nm}$ & $5.4 \mathrm{~mm}$ & $20 \mathrm{~V}$ & 30 \\
\hline Lenses & Condenser & ACL108 & $f 4.2 \mathrm{~mm}$ & $10 \mathrm{~mm}$ & & 16.8 \\
\hline Dichroic & Longpass & Edmund 87-051 & $\lambda 435 \mathrm{~nm}$ & $12.5 \mathrm{~mm}$ & & 140 \\
\hline \multicolumn{7}{|c|}{$>435 \mathrm{~nm}$ band } \\
\hline Optoelectronics & Photodiode & Thor FDS025 & $\lambda 400-1100 \mathrm{~nm}$ & $5.4 \mathrm{~mm}$ & $20 \mathrm{~V}$ & 30 \\
\hline Lenses & Condenser & ACL108 & $f 4.2 \mathrm{~mm}$ & $10 \mathrm{~mm}$ & & 16.8 \\
\hline \multicolumn{7}{|c|}{ Digitization } \\
\hline Computer & Tablet & Dell Venue 8 Pro & MATLAB ${ }^{\circledR}$ & $8 \mathrm{in.}$ & $5 \mathrm{~V}$ & 250 \\
\hline Oscilloscope & USB & Virtins 2820R & $200 \mathrm{MHz}$ & & $5 \mathrm{~V}$ & 250 \\
\hline
\end{tabular}


$\tau_{\phi}=\omega^{-1} \tan \Delta \phi$

and

$\tau_{m}=\omega^{-1}\left(m^{-2}-1\right)^{1 / 2}$

In this study, the characteristic lifetimes of collagens at three wavelength bands were quantified to provide the ability for spectral-lifetime discrimination.

\section{Method}

\subsection{Miniaturized Optics}

The system was based on free space optics with a common optical path for the excitation and the emission intensities to optimize coupling and increase signal (Fig. 2). It achieved spectrallifetime measurements by breaking up incoming intensity into three different bands via a series of longpass dichroics. Specifically, the bands were separated by longpass dichorics at 387,409 , and $435 \mathrm{~nm}$. The first two bands, referred to here on as the 400 and $420 \mathrm{~nm}$ bands at their center wavelengths, correlated to the fluorescence emission profiles of collagens I and III. A wavelength above $435 \mathrm{~nm}$, referred to here on as $>435 \mathrm{~nm}$ band, can help to remove noncollagen backgrounds.

First, the modulated LED light source was filtered by a 357nm, 44-nm bandpass filter and collected by an aspherical condenser lens to couple light toward the top surface of the first dichroic (387 nm, longpass). The 357-nm light was then reflected by dichroic toward the lens at the sample. This excitation was illuminated on a fluorescence sample at a fixed geometry dictated by the 3-D printed cavity and the lens' focal length. The resultant emission and scattering were collected reversely through the same lens geometry and filtered through the same dichroic, removing nonfluorescent scattering below $387 \mathrm{~nm}$. Similar to aforementioned LED/first dichroic stage, the emission light was directed onto two other dichroics at the prescribed longpass wavelengths. Each of the subsequent three wavelength bands was then focused by an aspherical condenser lens onto a fast, PIN photodiode. The complete list of optical and electronic components is described in Table 1.

\subsection{Custom Built Optomechanics}

To house the optical system, a tube was designed as two halves split down the center of its vertical orientation, with several joints added on to provide the mating surface [Fig. 3(a)]. The three dichroics dividing the system into four stages were designed with minimal distance from each other, allowing several millimeters for LED, heatsink, PIN photodiode alignment, and mounting only. The shorter component distances, common axis, and free space design of the micro-optics system ensure minimal coupling loss in a miniaturized package.

The coupling of the system can be interpreted by the optical power measured at various stages in the micro-optics system. A cellulose-based paper business card was used for coupling measurements due to its similarity in excitation/emission wavelengths as collagen and its strong fluorescence (Table 2). The power of the LED module going into the optics and the resultant power at the sample plane were measured as $I_{0}$ and $I_{\text {coupled }}$, respectively, for the excitation stage. The resultant fluorescence from the paper was then measured with a calibrated spectrometer at their appropriate wavelengths. The $I_{\text {coupled }}$ for each band was then measured after the appropriate dichroics and lenses, at the respective photodiode positions.

\subsection{Thermal and Mechanical Finite Element Analysis}

The design of the 3-D printed optomechanic system was evaluated by both thermal and mechanical analyses using a 3-D CAD model of the device and FEA. Initially, a 3-D model of the micro-optics system was constructed in CAD software (Inventor, Autodesk Inc.). The model was then discretized into a mesh geometry that was constrained and joined by nodes. The mesh model was then applied in the thermal and mechanical analyses with appropriate governing equations.

Table 2 Measured coupling at different stages of the micro-optics system.

\begin{tabular}{lccc} 
Stages & & $I_{0}(\mu \mathrm{W})$ & $I_{\text {Coupled }}(\mu \mathrm{W})$ \\
\hline Excitation & & 19.9 & 16.2 \\
Paper $^{\mathrm{a}}$ & $400 \mathrm{~nm}$ & 0.16 & 0.06 \\
& $420 \mathrm{~nm}$ & 0.12 & 0.05 \\
& $>430 \mathrm{~nm}$ & 0.11 & 0.90 \\
\hline
\end{tabular}

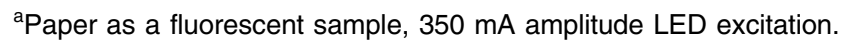

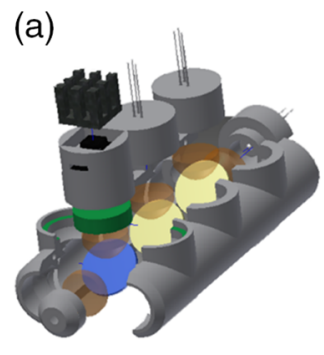

(b)

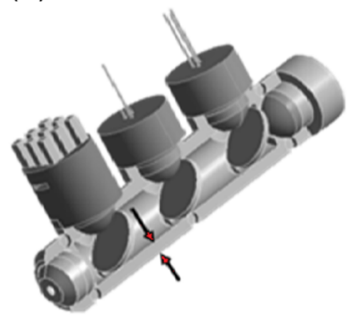

(c)

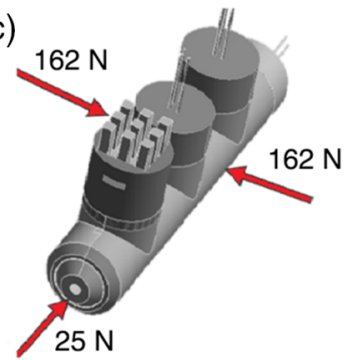

Fig. 3 3-D optomechanics system and FEA parameters. (a) The gray components represent the optical tube mounts and LED/photodiode adapters that were 3-D printed. The blue and gold colored filters represent the $1 / 2$ " longpass dichroics at prescribed wavelengths. The transparent orange shapes are $10-\mathrm{mm}$ condenser lenses to provide the focusing and collimation for each stage. The overall dimension of the tube, not including the adapters, is around $1.5 \mathrm{in} . \times 3 \mathrm{in}$. .. (b) Device thickness was varied in simulation. (c) Mechanical loading on device was modeled after normal gripping $(162 \mathrm{~N})$ and adequate contact on sample/skin $(25 \mathrm{~N})$. 
3-D thermal strain was computed by specifying the thermal properties of the material (such as specific heat and thermal conductivity) and applied conditions (such as heat flux and temperature boundary conditions). In addition to stress and strain, the 3-D temperature distribution was also obtained. FEA for both transient thermal and steady state thermal analyses was conducted using ANSYS. Similar to the thermal analysis, external mechanical loads from device handling were applied to the discretized finite element mesh in combination with defined material properties, to allow for numerical determination of strain at each elemental node and the stresses within each element. The deformations subsequent to both thermal and applied mechanical loading were determined for several combinations of structural and material considerations.

\subsection{Finite Element Analysis Parameters Investigated}

The primary heating load for the device came from the LED that generated the incident light prior to contacting the tissue. The LED was obtained from Digikey (537-1092-1-ND) capable of 1 A current at $4.1 \mathrm{~V}$. Based on our system coupling characterizations and typical tissue fluorescence emission (Table 2), we designed the LED with a maximum modulation of $200 \mathrm{~mA}$ to maintain photodiode intensities around a fraction of $\mu \mathrm{W}$. Since current LED efficiency peaked at $40 \%$ especially for blue wavelengths, ${ }^{42,43}$ the joule heating was estimated at $0.5 \mathrm{~W}$. Thus the analysis was carried out by applying a heating load of $0.5 \mathrm{~W}$ to all surfaces directly exposed to the LED. Various structural thicknesses were investigated using this thermal modeling [Fig. 3(b)].

Contrary to the single thermal load, the detection system was subject to multiple mechanical loads. The device is to be held by a clinician, which is translated into two forces applied perpendicularly to both sides of the device. Based on studies of handheld device in "Key Pinch" positions, ${ }^{44}$ right-handed males at 20 to 24 years of age had an average key pinch strength of $36.5 \mathrm{lbf}$ or $162 \mathrm{~N}$ (90th percentile of this demographic). Thus, we simulated a load of $162 \mathrm{~N}$ to both sides of the device [Fig. 3(c)]. Additionally, the device was designed to come into contact with the patient's body during normal operation. Therefore, it was assumed that the frontal load on the device would be no more than $15 \%$ of the gripping strength, or $25 \mathrm{~N}$ applied at the front of the device [Fig. 3(c)]. Various thicknesses were investigated using this mechanical loading model.

Our stereolithography technique is limited by its print material, unlike the array of available plastics in extrusionbased 3-D printing techniques. To investigate whether material choice is a factor in our printed geometry, we simulated the structure using three different materials-VisiJet ${ }^{\circledR}$ FTX Green, polylactic acid (PLA), and acrylonitrile butadiene styrene (ABS). VisiJet is the material used in our stereolithography printer, while PLA and ABS are available in extrusion-based 3$\mathrm{D}$ printers. Along with the variation of the material type, four wall thicknesses-2.54, 3.175, 3.81, and $4.45 \mathrm{~mm}$ (0.1 in., 0.125 in., 1.5 in., and 1.75 in.) - were also investigated to determine the optimal dimension for each material.

\subsection{Optical Ray Tracing}

The ray tracing program TracePro (Lambda Research) was used to model the light propagation in the device and demonstrate the feasibility of optical system. This was done on a geometry with the optimal wall thickness as characterized from the thermal and mechanical FEAs. Due to the common path of the fluorescence excitation and emission employed in this design, the ray tracing was broken into two parts-excitation and emission geometries. Only the geometric optics without scattering or multiple reflections were modeled. Four wavelengths - one excitation and three for each emission bands-were simulated separately in the ray tracing. The positions of the optics and the optimal material thickness from FEA were adjusted to optimize the ray tracing toward the smallest spot sizes to ensure maximum coupling.

\subsection{Frequency Domain Method}

The prototype was applied to the detection of collagen I and III samples (dried fiber from bovine origin obtained from Sigma Aldrich, catalog numbers C9879-1G and CC078 for type I and III, respectively). During data acquisition, the raw emission signals from the photodiodes were digitized by the USB oscilloscope. To remove nonlinear DC offsets from different scaling and frequencies of the raw signals, offsets from dark readings (LED turned off) at specific measurement conditions were subtracted from the raw data before fitting and data processing. This process allowed more accurate calculations of the phase shift angles and modulation depths, without peak uncertainties, amplitude drifts, and waveform asymmetries from one part of the raw data to another. Raw data of $2 \times 10^{4}$ points were acquired by the oscilloscope, segmented into 1250 points strings, and averaged together to provide a working data length. This self-averaging allowed for faster data acquisition while averaging over data jitters. FFT was done on this data length to extract the phase and modulation components at the particular frequency that was scanned. Frequencies at 10, 20, 30, 40, 50, and $60 \mathrm{MHz}$ were scanned, and 10 sets of frequency scans were done for each sample being measured. All of the data acquisition and signal analysis were done within MATLAB ${ }^{\circledR}$ (Mathworks, Inc.) with vendor supplied hardware drivers.

After the phase angles and modulation depth ratios were calculated from 10 through $60 \mathrm{MHz}$, the data were fitted to a multiexponential model, ${ }^{18,45}$ where the fluorescence impulse response function was described in Eq. (1). Thus, the fractional intensity observed in the usual steady-state measurement due to each component in the multiexponential decay ${ }^{29}$ is

$f=\frac{\alpha_{i} \tau_{i}}{\sum_{j} \alpha_{j} \tau_{j}}$

where $\sum f_{i}=1$. Lifetime can be finally calculated as

$\tau=\sum f_{i} \tau_{i}$.

\subsection{Electronics and Data Acquisition}

The optoelectronics and data acquisition of the system were designed to achieve optimal portability. A custom LED driver circuit and switchable photodiode transimpedance amplifier were initially designed for the micro-optics system. However, both prototype boards were too large at $1 \mathrm{in} . \times 3 \mathrm{in}$. dimensions and not suitable for integration. Therefore, the biophotonics results were finally obtained by guiding an LED source modulated at 10 to $60 \mathrm{MHz}$ (Thorlabs DC3100-365) to the microoptics system via a $0.22-\mathrm{NA} \quad 600-\mu \mathrm{m}$ UV solarized fiber 
cable (Stellarnet). Similarly, the emissions after each dichroic were manually guided to an avalanche photodiode (Thorlabs APD120A2) with $2.5 \times 10^{6} \mathrm{~V} / \mathrm{W}$ gain via $600-\mu \mathrm{m}$ fiberoptics.

Digitization of both the LED trigger signal and photodiode signal was provided by USB DSO (Virtins DSO-2820R). $2 \times 10^{4}$ sample data at $2 \mathrm{GS} / \mathrm{s}$ were acquired within the DSO's software. Raw data were imported into MATLAB ${ }^{\circledR}$ for FFT analysis and calculation of the fitted two exponential decay models as described earlier. All computer controls were performed on a Dell Venue 8 Pro tablet running Windows 10. The entire portable package, include the computing unit, battery, and micro-optics, weighed less than 4 pounds and can fit in a small suitcase (Fig. 1).

\section{Result and Discussion}

Thermal and mechanical FEA were conducted before the devices were printed. Both models showed negligible deformations, which did not lead to loss of optical performance. The geometry was further optimized with ray tracing to form the final 3-D printed structure. After calibration of the devices, lifetimes of type I and III collagens in the three wavelength bands were measured and calculated.

\subsection{Thermal Finite Element Analysis Results Indicate Negligible Strain}

As stated earlier, the thermal analysis was carried out with the assumption that all surfaces exposed to the LED underwent a heat flow of $0.5 \mathrm{~W}$. All temperature gradients were localized near the LED emitter, as the plastic material is expected to have low thermal conductivity. A temperature probe was placed on the cylinder surface below the LED emitter and its readings were plotted for the different materials in Fig. 4(a). During our modeling, temperature distributions remained within $2.54 \mathrm{~mm}$ of the wall thickness. Thus, thermal analyses were only carried out at a wall thickness of $2.54 \mathrm{~mm}$ for each material.

A clear difference in the materials' response to the heating load can be observed. Over a period of $60 \mathrm{~s}$, VisiJet ${ }^{\circledR}$ FTX Green was clearly the superior choice in terms of heat dissipation. Furthermore, ABS was the most inferior material with respect to thermal performance, reaching a temperature over $110^{\circ} \mathrm{C}$ after
$60 \mathrm{~s}$. The device is expected to be operated in short measurement durations, e.g., several seconds each for the three channels and dark noise readings, totaling up to $10 \mathrm{~s}$ or so of continuous operation. At these conditions, the PLA and VisiJet temperatures stayed below the typical scalding temperatures for contact, which would be reached at $60^{\circ} \mathrm{C}$ after $20 \mathrm{~s}$. Thus, by considering only the thermal characteristics, it is evident that PLA and VisiJet $^{\circledR}$ FTX Green were comparable options for the device's structural material. It should be noted that all materials showed negligible thermal strain for their respective maximum temperatures reached. Therefore, the thermal characteristics of various materials were only considered from an operator's safety and comfort perspective rather than the functional optomechanics perspective.

\subsection{Mechanical Finite Element Analysis Results are Comparable to Other 3-D Printings}

On the other hand, the device was subject to the mechanical loading conditions described in the FEA parameters section. The device experienced deformation along multiple axes during the simulation. However, only the deformation that occurred normal to the main optical axis of device appeared to be significant. Deformation along other axes of the device were minute and evenly distributed, so any lateral distortions to the optical mounts and dichroic were negligible. To compare the various materials, the maximum interior deformations of the device were plotted for various wall thicknesses in Fig. 4(b). All three materials observed the same amount of deformation for wall thicknesses of 2.54 and $3.175 \mathrm{~mm}$. When the wall thickness increased beyond $3.175 \mathrm{~mm}$, a noticeable difference across the various materials occurred. Notably, all materials had deformation that leveled out at thickness above $3.81 \mathrm{~mm}$. Based on lowest stress-strain curve, PLA was the ideal choice for the structural components, followed by ABS and then VisiJet ${ }^{\circledR}$ FTX Green. In addition, $3.81 \mathrm{~mm}$ was the optimal thickness for PLA and ABS, while no improvements were observed for VisiJet with thickness above $3.175 \mathrm{~mm}$ for the designed optomechanics geometry.

Post-FEA, all subsequent designs and builds using our Projet 1200 stereolithography printer adopted a 3.175 -mm thickness.
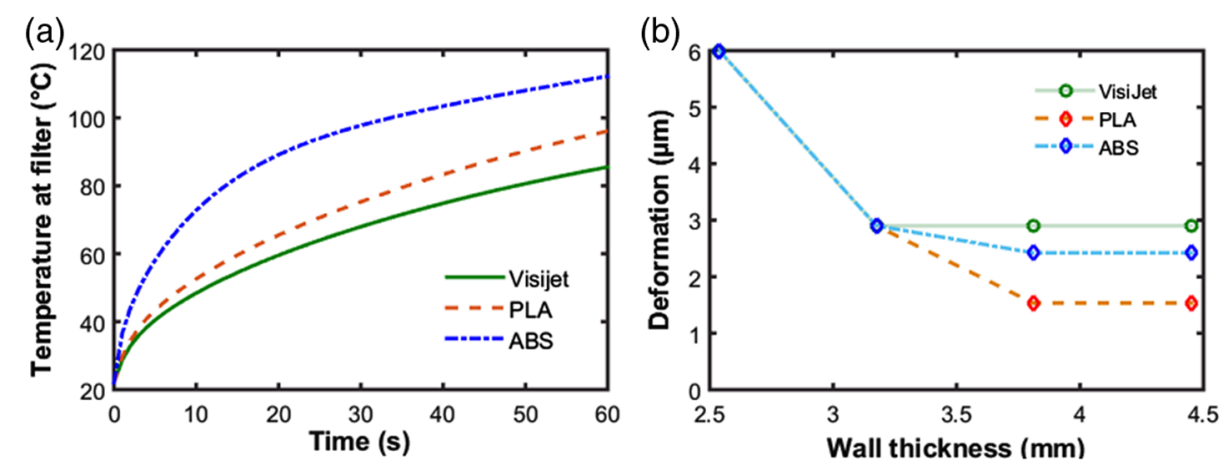

Fig. 4 Thermal and mechanical FEA for wall thickness and materials in 3-D printing. To compare the materials between stereolithography and extrusion-based 3-D printing, ABS, PLA, and VisiJet Green (3$D$ Systems) were investigated for thermal distribution and mechanical deformation in the designed geometry. (a) Temperature gradient localized around the LED emitter showed that ABS developed higher temperatures than PLA or VisiJet Green. Temperature measured in VisiJet material remained below $60^{\circ} \mathrm{C}$ after $20 \mathrm{~s}$ of typical LED operation. (b) Maximum deformation inside the micro-optics housing is plotted at different wall thicknesses for the three printing materials. All materials have similar deformations below $3.175 \mathrm{~mm}$, while ABS maintains the lowest deformation at higher wall thicknesses. 
(a)

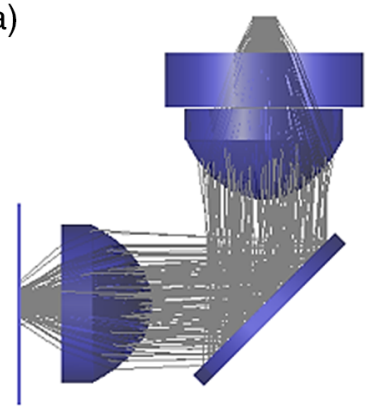

(b)

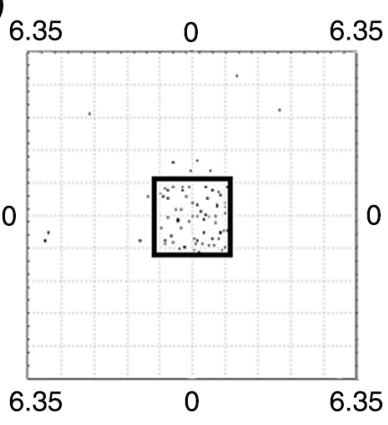

Fig. 5 Optical ray tracing simulation of the excitation stage. Excitation ray tracing is simulated from the LED source through filter, lenses, dicroic, and finally the sample. (a) Profile view of the ray tracing for the excitation stage. (b) Total irradiance map on the surface of sample. The light spot is around $2.6 \mathrm{~mm} \times 2.6 \mathrm{~mm}$, which is close to the size of LED light source of $2.3 \mathrm{~mm}$, represented by the square box.

\subsection{Ray Tracing for Optical Optimization}

At the optimal wall thickness modeled in the thermal and structural analyses of the photonic reader device, there were no observable effects upon the ray tracing performance of the device. The deformations of the housing and the subsequent relative motions of the optical components still resulted in appropriate optical quality and performance, as simulated through the optical ray tracing. Furthermore, to ensure minimal coupling losses, the distances between parts were optimized to produce ray tracing with the smallest spot sizes.

Ray tracing of the excitation stage was modeled separately from the rest of the system to simplify the common axis for fluorescence in the optomechanical design [Fig. 5(a)]. A 365-nm light from the LED passed through an excitation filter and

a condenser lens, reflected from a 45-deg dichroic, and was focused on a sample through a second condenser lens. Due to the finite size of the LED emitter $(2.3 \mathrm{~mm})$, its image at the sample was adjusted relative to the focal length of the condenser lens in order to achieve a focused spot of $2.6 \mathrm{~mm}$ [Fig. 5(b)].

Next, the emission stages corresponding to the three detection bands were modeled in ray tracing using 400,420 , and $435 \mathrm{~nm}$ wavelengths [Fig. 6(a)]. The resultant intensity showed spot sizes of $1.8,1.5$, and $1.5 \mathrm{~mm}$ diameters at the detector planes of the 400,420 , and $>435 \mathrm{~nm}$ stages, respectively [Fig. 6(B)]. After the ray tracing analysis, the CAD design was updated for 3-D printing. The final 3-D printed geometry represented the optimized structure that provided the smallest spot size via ray tracing.

\subsection{Frequency Domain Phase Shift Results}

For both collagen samples measured, the phase shift angles rose with increasing frequency with an inverse tangent profile, Figs. 7(a) and 7(b), consistent with Eq. (2). Higher phase shift denotes to longer lifetime at a particular frequency. Upon closer examination of the differences between the three channels, the first channel always resulted in the lowest phase shift. At higher frequencies, 420-nm band phase shift rose above that of the $>435 \mathrm{~nm}$ band for collagen I, but the two bands overlapped across frequencies for collagen type III.

\subsection{Frequency Domain Demodulation Results}

For both collagen samples measured, the modulation depth ratios decreased with increasing frequency following a decay profile [Figs. 7(c) and 7(d)], which was consistent with Eq. (3). At a particular frequency, deeper demodulation generally denotes a longer lifetime. As a trend, collagen I had

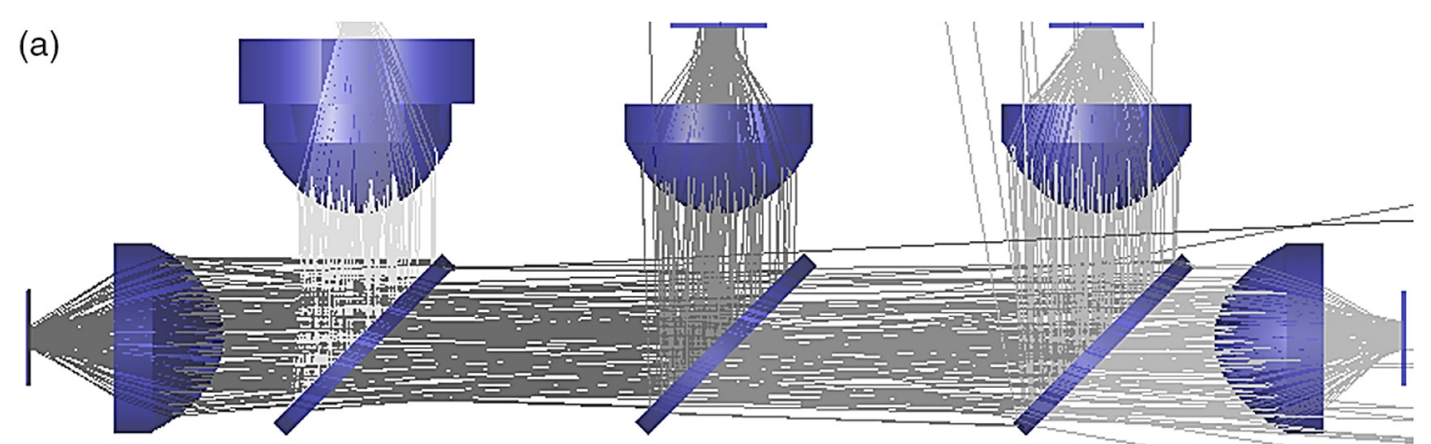

(b)

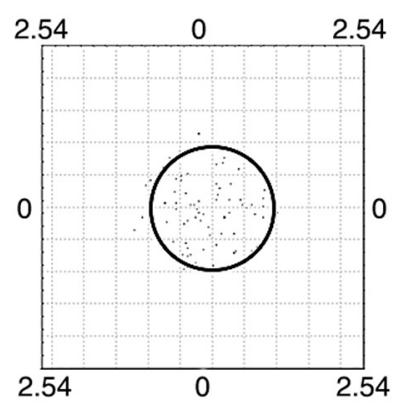

(c)

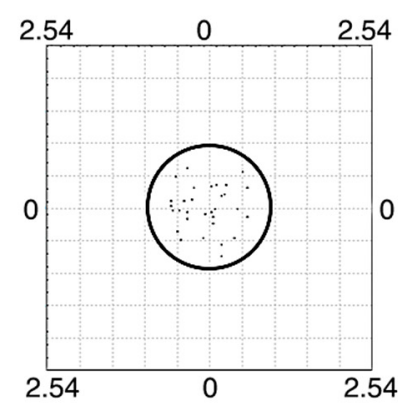

(d)

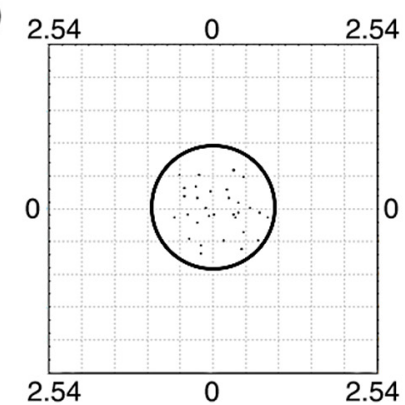

Fig. 6 Optical ray tracing simulation of the emission stages. Emission ray tracing is simulated from the sample, through the optical system and to target photodiodes for each detection band. (a) Profile of ray tracing with the diameter of fluorescence light source at $2.6 \mathrm{~mm}$. (b-d) represent the total radiance on photodiodes, which have size of $1.8,1.5$, and $1.5 \mathrm{~mm}$, respectively. The circles provide references to the actual photodiode sensor area. 

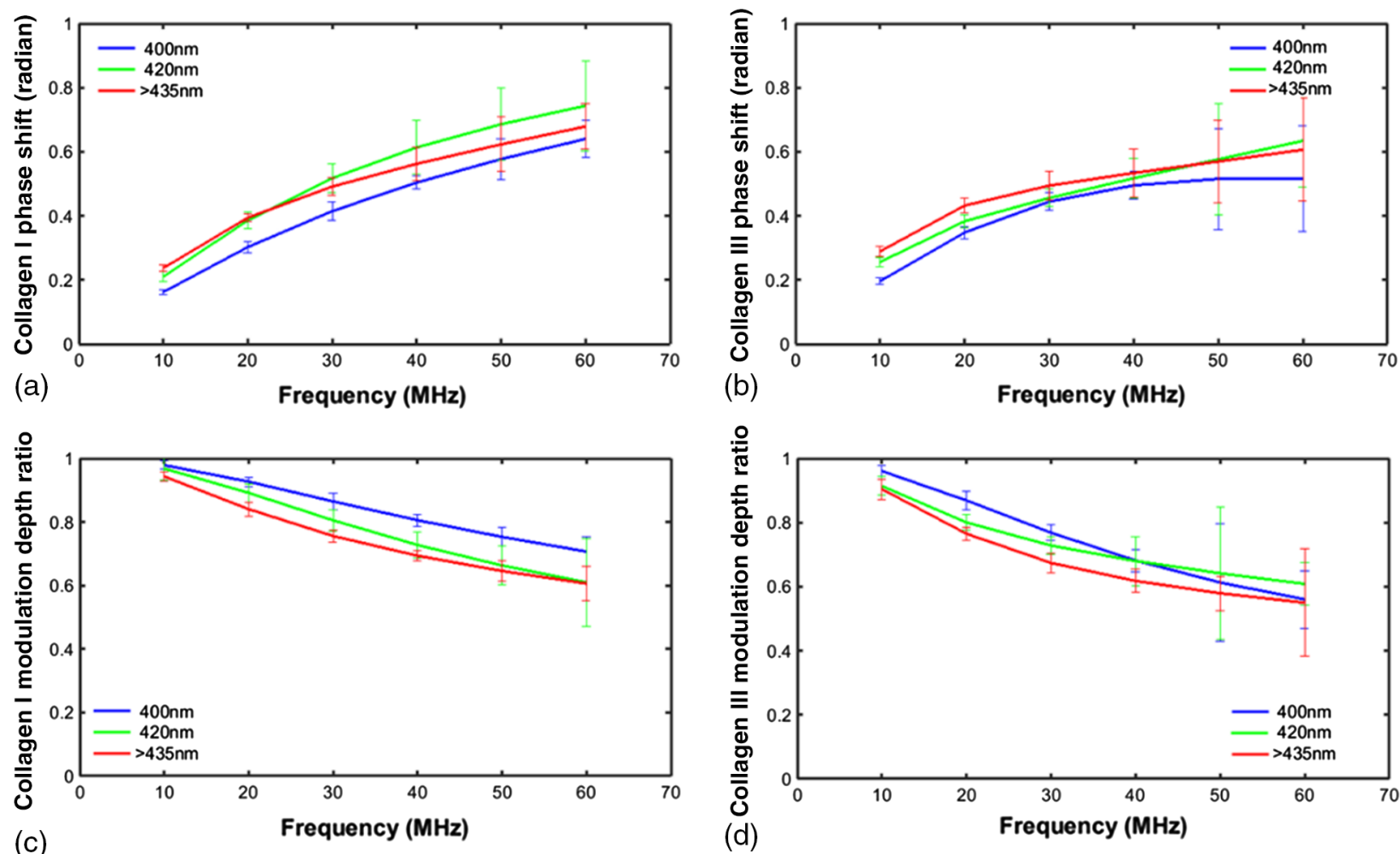

Fig. 7 Frequency domain results for spectral-fluorescence separation of collagen I/III. Collagen I results are shown in the left hand column ( $a$ and $c$ ), while collagen III results are shown in the right (b and d). For both the phase (top row) and modulation (bottom row) graphs, the 400, 420, and $>435 \mathrm{~nm}$ bands are plotted as blue, green, and red, respectively. $400 \mathrm{~nm}$ bands showed phase and modulation results consistent with shorter lifetimes for both types of collagen. Phase results for collagen I, (a), are smaller than that of collagen III, (b), but collagen III flattens out above $30 \mathrm{MHz}$. Similarly, modulation results for collagen I, (c), are shallower than that of collagen III, (d). Generally, bands for collagen type I are further separated, while collagen type III has more overlaps and crossovers.

shallower demodulation than collagen III. Specifically, modulation depth ratios showed more separation between 400 and $435 \mathrm{~nm}$ bands, as the measurements showed consistent, smaller standard deviations.

\subsection{Frequency Domain Lifetime}

To differentiate the lifetimes of type I versus type III collagen for the three spectral channels, phase and demodulation data were fitted in a two-exponential decay model and tabulated in Table 3.
In the table, $\tau_{\varphi}$ is the phase shift lifetime, $\tau_{m}$ is the demodulation lifetime, and $\tau$ is the apparent lifetime. Apparent lifetimes were calculated using a minimization function that weighed both phase and demodulation data according to their standard deviations. Spectral-lifetime results in Table 3, which ranged between 2 and 4 ns for type I and 3 and 5 ns for type III, compared well with lifetime measurements from other collagen autofluorescence reports. ${ }^{16-18,46,47}$

Lifetimes increased from $400 \mathrm{~nm}$, to $420 \mathrm{~nm}$, and to above $435 \mathrm{~nm}$ for both collagens. Collagen III had a longer lifetime

Table 3 Calculated lifetimes for collagen I and III at multiple bands.

\begin{tabular}{|c|c|c|c|c|c|}
\hline Type & Lifetime & $400 \mathrm{~nm}$ & $420 \mathrm{~nm}$ & $>435 \mathrm{~nm}$ & TD Ref. \\
\hline \multirow[t]{3}{*}{ Col I } & $\tau_{\varphi}(\mathrm{ns})$ & $2.2767 \pm 0.0442$ & $2.9151 \pm 0.0516$ & $2.8211 \pm 0.0430$ & \\
\hline & $\tau_{m}(\mathrm{~ns})$ & $3.0007 \pm 0.1769$ & $3.8135 \pm 0.2552$ & $4.4541 \pm 0.0955$ & \\
\hline & $\tau(\mathrm{ns})^{\mathrm{a}}$ & $2.6484 \pm 0.0599$ & $3.4559 \pm 0.1116$ & $4.1087 \pm 0.1310$ & $\sim 3 \mathrm{~ns}^{16-18,46,47}$ \\
\hline \multirow[t]{3}{*}{ Col III } & $\tau_{\varphi}(\mathrm{ns})$ & $2.3380 \pm 0.0926$ & $2.7091 \pm 0.1024$ & $2.9145 \pm 0.0544$ & \\
\hline & $\tau_{m}(\mathrm{~ns})$ & $4.3116 \pm 0.3625$ & $4.9300 \pm 0.1649$ & $5.6041 \pm 0.1655$ & \\
\hline & $\tau(\mathrm{ns})^{\mathrm{a}}$ & $3.2600 \pm 0.1462$ & $4.8088 \pm 0.1954$ & $5.3316 \pm 0.1626$ & $\sim 4 \mathrm{~ns}^{16-18,46,47}$ \\
\hline
\end{tabular}

Note: Time domain (TD) references provided for comparison.

${ }^{a} A p p a r e n t$ lifetimes via two-exponential fitting. 
than collagen I. In our results, both spectral intensities and lifetimes contribute to the separation of the collagen I versus III. A 400-nm band had higher signal intensities in general, but smaller lifetime differences between the collagen types, while 420-nm band had lower signal intensities but greater lifetime differences. In the application of wound healing monitoring, where closed wound undergoes collagen remodeling from type III to type I collagens, ${ }^{2,3}$ the emission intensity will be reduced, and 400$\mathrm{nm}$ band should provide an important measurement due to its higher intensity. However, 420 -nm band should provide a better lifetime contrast despite its lower intensity. By leveraging both channels for spectral-lifetime detection, more precise differentiation, and thus accurate monitoring of tissue collagen can be achieved.

\section{Future Works}

Despite efforts to miniaturize the optomechanics structure, the prototype LED driver and photodiode transimpedance amplifier circuitry still required significant space. Additional embedded solutions are needed to provide further electronics integration. Moreover, data acquisition was done manually with DSO's software and imported into MATLAB ${ }^{\circledR}$. A simple graphical user interface should make system operation user-friendly and less prone to errors. Additional structural mechanics such as the handle housing and wiring have yet to be implemented as well. The low intensity in actual tissue applications may require the use of avalanche photodiodes, which require higher bias voltages. Finally, multiple excitation wavelengths may be possible in the same optical train by the use of multicolored LED diodes, which may broaden the available tissue fluorophores to detect, e.g., nicotinamide adenine dinucleotide.

The collagens used in this article are purified fibrous proteins and intended as a proof of concept of the miniaturized probe. We have noticed different sources of proteins, e.g., skin and tendon, as well as different preparations and solutions greatly affect collagen autofluorescence lifetimes. ${ }^{17,18,47,48}$ An extensive tissuebased study is necessary to provide confidence in the frequency-domain technique as a diagnostic tool, similar to efforts in time-domain studies. ${ }^{48}$ In clinical applications, we envision the probe to be calibrated for individual patient's baselines, e.g., a person's normal skin versus healing wound. The relative differences of tissue collagens for that patient will then be the measurement instead of an absolute lifetime value, where the dynamics of the measurements over time provide diagnostic information rather than static snapshots.

In this study, the miniature spectra-lifetime system was applied to the detection of type I versus III collagen, which are the major parameters of tissue remodeling in a closed wound. In the future, such portable spectral-lifetime system can be applied to clinical detection of advanced glycation end-products in diabetic skin, tissue fibrosis, and scarring in wounds, and collagen/ elastin compositions in aging skin, among others.

\section{Conclusion}

A miniaturized optical system was designed for spectral-lifetime measurements of collagens I and III. The system was comprised of a small tablet and USB DSO, LED, photodiode elements, and a 3-D printed optomechanic structure, well-suited to portable detection applications. Thermal and mechanical FEA demonstrated negligible deformations during expected operation and handling of the device. Ray tracing further optimized the 3-D printed optomechanics structure. Type I and III collagen lifetimes were measured across three spectral bands with our device and were distinguished from each other. We found that $400 \mathrm{~nm}$ band gave higher signal intensity while the $420 \mathrm{~nm}$ band provided greater lifetime differences between collagen I and III. In tissue applications where low autofluorescence is combined with minute differences in collagen composition, multiple spectral channels with lifetime measurements may allow better collagen detections than either one along. The 3-D prototyped miniature system has the potential to provide clinicians with a useful yet simple to use device for tissue collagen detections.

\section{Acknowledgments}

This work was funded by the University of Michigan-Dearborn Institute for Advanced Vehicle Systems (IAVS) seed fund and by the Department of Mechanical Engineering (undergraduate support).

\section{References}

1. I. A. Darby and T. D. Hewitson, "Fibroblast differentiation in wound healing and fibrosis," Int. Rev. Cytol. 257, 143-179 (2007).

2. H. P. Ehrlich, "The role of connective tissue matrix in wound healing," Prog. Clin. Biol. Res. 266, 243-258 (1988).

3. M. J. Ranzer, L. Chen, and L. A. DiPietro, "Fibroblast function and wound breaking strength are impaired by acute ethanol intoxication," Alcohol Clin. Exp. Res. 35, 83-90 (2011).

4. J. F. Almine, S. G. Wise, and A. S. Weiss, "Elastin signaling in wound repair," Birth Defects Res. Part C: Embryo Today: Rev. 96(3), 248-257 (2012).

5. S. Katsuda et al., "Collagen in human atherosclerosis: immunohistomchemical analysis using collagen types-specific antibodies," Arterioscler. Thromb., Vasc. Biol. 12, 494-502 (1992).

6. L. C. U. Junquiera, G. Bignolas, and R. R. Brentani, "Picrosirius staining plus polarization microscopy, a specific method for collagen detection in tissue sections," Histochem. J. 11, 447-455 (1979).

7. L. Chen et al., "Blockage of mast cell activation reduces cutaneous scar formation," PLoS One 9(1), e85226 (2014).

8. J. F. Lo et al., "Microfluidic wound bandage: localized oxygen modulation of collagen maturation," Wound Repair Regener. 21(2), 226-234 (2013).

9. M. J. Koehler et al., "In vivo assessment of human skin aging by multiphoton laser scanning tomography," Opt. Lett. 31(19), 2879-2881 (2006).

10. W. R. Zipfel et al., "Live tissue intrinsic emission microscopy using multiphoton-excited native fluorescence and second harmonic generation," Proc. Natl. Acad. Sci. U. S. A. 100(12), 7075-7080 (2003).

11. L. Brancaleon et al., "Monitoring photoaging by use of multiphoton fluorescence and second harmonic generation microscopy," Proc. SPIE 6078, 607803 (2006).

12. J. Adur et al., "The severity of Osteogenesis imperfecta and type I collagen pattern in human skin as determined by nonlinear microscopy: proof of principle of a diagnostic method," PLoS One 8(7), e69186 (2013).

13. P. Steven et al., "Comparison of cornea module and dermaInspect for noninvasive imaging of ocular surface pathologies," J. Biomed. Opt. 14(6), 064040 (2009).

14. P. Steven et al., "Imaging corneal crosslinking by autofluorescence 2photon microscopy, second harmonic generation, and fluorescence lifetime measurements," J. Cataract Refract. Surg. 36(12), 2150-2159 (2010).

15. G. Deka, W. Wu, and F. Kao, "In vivo wound healing diagnosis with second harmonic and fluorescence lifetime imaging," J. Biomed. Opt. 18(6), 061222 (2013).

16. P. Ashjian et al., "Noninvasive in situ evaluation of osteogenic differentiation by time-resolved laser-induced fluorescence spectroscopy," Tissue Eng. 10(3-4), 411-420 (2004).

17. L. Marcu et al., "Characterization of type I, I, I, IV, and V collagens by time-resolved laser-induced fluorescence spectroscopy," Proc. SPIE 3917, 93 (2000). 
18. R. Liu et al., "Compact, non-invasive frequency domain lifetime differentiation of collagens and elastin," Sens. Actuators, B 219, 283-293 (2015).

19. Z. Deyl et al., "Studies on the chemical nature of elastin fluorescence," Biochim. Biophys. Acta 625(2), 248-254 (1980).

20. D. R. Eyre, M. A. Paz, and P. M. Gallop, "Cross-linking in collagen and elastin," Annu. Rev. Biochem. 53, 717-748 (1984).

21. E. Fujimori, "Changes induced by ozone and ultraviolet light in type I collagen: bovine Achilles tendon collagen versus rat tail tendon collagen,” Eur. J. Biochem. 152, 299-306 (1985).

22. O. Vančíková and Z. Deyl, "Disappearance of tyrosine residues in collagen with age. Suggestion of a possible reaction mechanism," Exp. Gerontol. 9(3), 123-130 (1974).

23. I. Miksík and Z. Deyl, "Change in the amount of epsilon-hexosyllysine, UV absorbance, and fluorescence of collagen with age in different animal species," J. Gerontol. 46(3), B111-B116 (1991).

24. V. M. Monnier, R. R. Kohn, and A. Cerami, "Accelerated age-related browning of human collagen in diabetes mellitus," Proc. Natl. Acad. Sci. U. S. A. 81(2), 583-587 (1984).

25. R. Meerwaldt et al., "Simple noninvasive measurement of skin autofluorescence," Ann. N. Y. Acad. Sci. 1043, 290-298 (2005).

26. N. Krstajić et al., " $256 \times 2$ SPAD line sensor for time resolved fluorescence spectroscopy," Opt. Express 23(5), 5653-5669 (2015).

27. D. K. Bird et al., "Simultaneous two-photon spectral and lifetime fluorescence microscopy," Appl. Opt. 43(27), 5173-5182 (2004).

28. P. P. Provenzano et al., "Nonlinear optical imaging and spectral-lifetime computational analysis of endogenous and exogenous fluorophores in breast cancer," J. Biomed. Opt. 13(3), 031220 (2008).

29. J. R. Lakowicz, Principles of Spectroscopy, 3rd ed., Springer, New York (2007).

30. C. Zhang et al., "Open-source 3D-printable optics equipment," PLoS One 8(3), e59840 (2013).

31. J. Wang et al., "Three-dimensional printing of tissue phantoms for biophotonic imaging," Opt. Lett. 39(10), 3010 (2014).

32. B. Berg et al., "Cellphone-based hand-held microplate reader for pointof-care testing of enzyme-linked immunosorbent assays," ACS Nano 9(8), 7857-7866 (2015).

33. A. Forcucci et al., "All-plastic, miniature, digital fluorescence microscope for three part white blood cell differential measurements at the point of care," Biomed. Opt. Express 6(11), 4433-4446 (2015).

34. B. C. Gross et al., "Evaluation of 3D printing and its potential impact on biotechnology and the chemical sciences," Anal. Chem. 86(7), 32403253 (2014).

35. D. Elson et al., "Time-domain fluorescence lifetime imaging applied to biological tissue," Photochem. Photobiol. Sci. 3, 795-801 (2004).

36. K. Dowling et al., "Fluorescence lifetime imaging with picosecond resolution for biomedical applications," Opt. Lett. 23(10), 810-812 (1998).

37. M. S. Patterson et al., "Frequency-domain reflectance for the determination of the scattering and absorption properties of tissue," Appl. Opt. 30(31), 4474-4476 (1991).
38. J. Mizeret et al., "Endoscopic tissue characterization by frequencydomain fluorescence lifetime imaging (FD-FLIM)," Laser Med. Sci. 12(3), 209-217 (1997).

39. A. D. Elder et al., "Calibration of a wide-field frequency-domain fluorescence lifetime microscopy system using light emitting diodes as light source," J. Microsc. 224, 166-180 (2006).

40. B. Yuan et al., "A cost-efficient frequency domain fluorescence lifetime measurement system," Am. J. Phys. 78(1), 28-34 (2010).

41. W. J. O'Hagan et al., "MHz LED source for nanosecond fluorescence sensing," Meas. Sci. Technol. 13, 84-91 (2002).

42. Y. Narukawa et al., "Ultra-high efficiency white light emitting diodes," Jpn. J. Appl. Phys. 45(2), 37-41 (2006).

43. M. R. Krames et al., "Status and future of high-power light-emitting diodes for solid-state lighting," J. Display Technol. 3(2), 160-175 (2007).

44. V. Mathiowetz et al., "Grip and pinch strength: normative data for adults," Arch. Phys. Med. Rehabil. 66(2), 69-74 (1985).

45. J. R. Lakowizc et al., "Analysis of fluorescence decay kinetics from variable-frequency phase shift and modulation data," Biophys. J. 46, 463477 (1984).

46. M. Y. Berezin and S. Achilefu, "Fluorescence lifetime measurements and biological imaging," Chem. Rev. 110(5), 2641-2684 (2010).

47. J. E. Phipps et al., "A fluorescence lifetime imaging classification method to investigate the collagen to lipid ratio in fibrous caps of atherosclerotic plaque," Lasers Surg. Med. 44(7), 564-571 (2012).

48. S. Ranjit et al., "Imaging fibrosis and separating collagens using second harmonic generation and phasor approach to fluorescence lifetime imaging," Sci. Rep. 5, 13378 (2015).

Luwei Zou is currently pursuing her master's degree in the Department of Mechanical Engineering, University of Michigan-Dearborn. Her research interests are applied mechanics and biophotonics.

Rui Liu is a mechanical engineer at Fiat Chrysler Automobiles. He received his MS degree in mechanical engineering from the University of Michigan-Dearborn (2015). His expertise includes automotive engineering; mechanical systems design, optimization, and integrations; optical systems design and testing, and biophotonics instrumentations.

Joe Fujiou Lo is an assistant professor of mechanical engineering at the University of Michigan-Dearborn. He received his BS degree in bioengineering from the University of California-Berkeley (2000) and $\mathrm{PhD}$ in biomedical engineering from the University of Southern California (2007). His postdoctoral experience includes ETH Zürich and University of Illinois-Chicago with expertise in microfluidic and microelectromechanical systems for biophotonic applications. The theme of his research is evolving traditional microengineering toward novel, multifaceted solutions for biomedical applications.

Biographies for the other authors are not available. 